\title{
Copaiba Oil
}

National Cancer Institute

\section{Source}

National Cancer Institute. Copaiba Oil. NCI Thesaurus. Code C72191.

The resinous oil tapped from the trunks of Copaifera reticulata. Copaiba oil is used medicinally for its ability to modulate the inflammatory process. 